\title{
Schopenhauer und Kant. Rezeption der „transzendentalen Analytik“ der Kritik der reinen Vernunft in der Frühphilosophie Schopenhauers
}

\author{
Yasuo Kamata \\ Kwansei Gakuin Universität - Japan
}

\section{1. Überblick: Schopenhauer und Kant}

Die Entstehung der Schopenhauerschen Philosophie verlief nicht eingleisig. Sie kann verstanden werden als eine Zusammensetzung und wechselseitige Beeinflussung verschiedener Ebenen, wie beispielsweise der Rezeption von und mit Kant, Fichte, Schelling und deren Zeitgenossen, den englischen Empiristen und den kontinentalen Rationalisten, der mittelalterlichen Scholastik und Mystik bis hin $\mathrm{zu}$ antiken Philosophen wie Platon und letztlich auch altindischer Philosophen. Hierzu gibt es heute zahlreiche Untersuchungen.

In seinem Hauptwerk Die Welt als Wille und Vorstellung nennt Schopenhauer seine erste Schrift, namentlich Ueber die vierfache Wurzel des Satzes vom zureichenden Grunde von 1813, eine Voraussetzung zum Verständnis seines Werkes. Diese erste Veröffentlichung Schopenhauers war durchaus im Geiste der Kantischen Philosophie verfaßt, wie wir im folgenden sehen werden.

Ohne Zweifel: Die oben genannten Philosophen und philosophischen Strömungen haben Schopenhauer kleine und große Denkanstöße gegeben, von denen er Platon und die alten Inder besonders hervorhebt, aber wir wissen heute auch, dass seine älteren Zeitgenossen wie Fichte und Schelling trotz Schopenhauers eigener oft negativer Bewertungen ihnen gegenüber, von diesen nicht minder beeinflußt wurde. Dennoch waren diese für die Grundlegung der Schopenhauerschen Philosophie von sekundärer Bedeutung, da die philosophische Grundlegung in Uber die vierfache Wurzel sich auf der transzendental philosophischen Ebene vollzieht, insofern die Frage nach der Bedingung der Möglichkeit der Erfahrung hier in das Zentrum rückt. Seinen vielseitigen Interessen entsprechend, ließ sich Schopenhauer zwar von vielen Philosophen und philosophischen Richtungen Denkanstöße geben, beispielsweise Schelling in der Naturphilosophie, Platon in der Ästhetik, die alten Inder in der Ethik, der rote Faden jedoch, an dem die Entstehung der Schopenhauerschen Philosophie im 
Hinblick auf deren philosophische Grundlegung nachverfolgt werden kann, ist jedoch die Kantische Philosophie. An Kants gedanklicher Strenge und Präzision hat Schopenhauer die Stringenz seiner Philosophie stets gemessen, auch wenn er sich nicht immer und mit jedem einzelnen Gedankengang Kants einverstanden erklärte. So konnte Schopenhauer im hohen Alter mit Genugtuung zurückblickend seine Philosophie als das „Zu-Ende-Denken der Kantischen Philosophie“ bezeichnen.

Wenn Schopenhauer nun schreibt, dass der Wille sich in der Welt als Vorstellung objektiviert, dann darf man diese Behauptungen nicht kritiklos hinnehmen wie ein religiöses Dogma und einfach repetieren in der Art „Nach Schopenhauer ist die Welt als Vorstellung die Objektivation des Willens“, sondern man muss stets fragen, was heißt das?, warum?, mit welcher Berechtigung?, mit welcher Stringenz des Denkens wird diese Behauptung begründet? Auch wenn in der Schopenhauerschen Philosophie solch einem Frageansatz im Sinne eines bloßen Hilfsmittel $(\mu \varepsilon \chi \alpha v \eta)$ des Willens der Primat abgesprochen werden sollte, so können wir dennoch nur mittels dieses Denkens die Unzulänglichkeit des Denkens selber konstatieren. Philosophie als solche ist ein verhängnisvolles Unternehmen des Denkens, insofern es nichts unhinterfragt sein lassen darf

So stellt meine heutige Ausführung in gewisser Weise ein Experiment dar, das Experiment der Frage nachzugehen, wie weit und wie konsequent man Schopenhauersche Philosophie im Spiegel der Kantischen Philosophie rekonstruieren kann, um dann eventuell zu sehen, mit welcher Stringenz er seine Philosophie aufgebaut hat. Dieses Experiment schliesst natürlich andere Experimente nicht aus, beispielsweise die strenge philosophische Integrität der Schopenhauerschen Philosophie aus anderer Perspektive wie der kritischen Rezeption von Fichte, Schelling oder eben anderen Philosophen und philosophischen Strömungen zu rekonstruieren. Im Gegenteil, nur durch solch eine Art und Weise von quasi paralleler Rekonstruktion wird der Blick auf die komplexe Entstehungsgeschichte der Schopenhauerschen Philosophie frei.

Durch die beschriebene sorgfältige Rekonstruktion anhand des Jünglingsmanuskriptes und seiner ersten Publikation Ueber die vierfache Wurzel wird Schopenhauers Anlehnung an Kant offenbar hinsichtlich der beiden Grundbegriffe der Schopenhauerschen Philosophie:Vorstellung und Wille.

Hierzu hebe ich die nachstehenden vier Punkte besonders hervor: 
1) Von der Kantischen „transzendentalen Deduktion der reinen Verstandesbegriffe“, insbesondere von dessen Gedanken der ursprünglichsynthetischen Einheit der Apperzeption gewinnt der junge Schopenhauer seinen Grundbegriff „der Welt als Vorstellung“. Schopenhauer zeigt in Ueber die vierfache Wurzel, dass die Erfahrung in der Korrelation der unmittelbaren Gegenwart der je einzelnen deutlichen Vorstellung im Selbstbewußtsein, genauer: im inneren Sinn („Vorgestelltwerden $\kappa \alpha \tau^{\prime} \quad \varepsilon v \tau \varepsilon \lambda \varepsilon \chi \varepsilon \varepsilon \alpha \nu^{\circ}$ ) und der Gesammtvorstellung, d.h. der potentiellen Vorstellung von dem Ganzen der Erfahrung als Hintergrund der unmittelbar gegenwärtigen deutlichen Vorstellung („Vorgestelltwerden $\left.\kappa \alpha \tau \alpha \quad \delta v v \alpha \mu v^{\star c}\right)$ besteht. Diese letztere, die inaktuale Gesammtvorstellung ist nämlich das, was die empirisch-objektive Realität des Erfahrungsganzen gewährleistet und was wir so im Alltag die objektive reale Welt nennen. (Go §18)

2) Von der Spontaneität der Apperzeption gewinnt der junge Schopenhauer die Grundkonzeption des Willens, sofern er hier die Bedingung der Möglichkeit der Erfahrung ausmacht. Diesen Willen möchte ich den transzendentalen Willen nennen, um ihn von demjenigen, sich innerweltlich objektivierenden Willen, zu unterscheiden, dem man innerhalb der bereits feststehenden Welt als Vorstellung begegnet, d.h. insofern deren empirisch-objektive Realität gesichert und vorausgesetzt ist. Dieser zweite Wille ist es, der z.B. in dem 2. Buch von Die Welt als Wille und Vorstellung als Wille in der Natur als deren Ding an sich, oder in dem 4. Buch hinsichtlich dessen „Loos in der sein Wesen abspiegelnden Welt“ (WI, §60) als Quelle des Leidens (WI, §52 etc.) angesprochen wird.

Nach Sichtung von Schopenhauers Studienheften gelangt man zu dem Schluß, dass ihm der Gedanke der Gleichsetzung von Spontaneität und Wille durch die Lektüre von Fichtes Versuch einer Critik aller Offenbarungen (1792) und System der Sittenlehre (1798) gekommen ist. Doch Schopenhauer distanziert sich von der Fichteschen spekulativen Erweiterung dieser Gleichsetzung. Stattdessen führt er die Gleichsetzung von Spontaneität und Wille auf Kant zurück und versucht den Willen auf der transzendentalkritischen Ebene lediglich als die Bedingung der Möglichkeit der Erfahrung darzustellen. Der transzendentale Wille wirkt als Spontaneität des Erkennens, die die Einbildungskraft initiiert, welche jedem menschlichen Erkenntnisvorgang zugrundeliegt. Die Platonische Idee wird nämlich auf der ursprünglichen, transzendentalphilosophischen Ebene als ethic@-Florianópolis, v. 11, n. 1, p. 54-67, julho de 2012. 
„Phantasma in Gegenwart der Vernunft“, d.h. als Produkt der Einbildungskraft definiert, und eben deshalb kann der Künstler als Genie die Platonische Idee durch seine Einbildungskraft antizipieren, vorwegnehmen und in den Kunstwerken konkretisieren, auch wenn die Natur die ideale Schönheit nicht oder noch nicht hervorbringen konnte. - Dieser alles entscheidende, transzendentalphilosophische Aspekt der Platonischen Idee in der Kunsttheorie Schopenhauers, die durch seine kritische Rezeption vor allem der Kritik der Urteilskraft entstanden ist, wird leider auch heute noch nicht ausreichend berücksichtigt.

3) Von Kants „Analytik der Grundsätze“ nimmt Schopenhauer nun die Bestimmung der oben genannten beiden Vorstellungsweisen her, indem die einzelne Vorstellung einerseits im Hinblick auf ihre intensive Größe als dem inneren Sinn unmittelbar gegenwärtige, deutliche Vorstellung ist, und andererseits im Hinblick auf ihre extensive Größe als die das ganze der Erfahrung ausmachende Vorstellung dargestellt. Auf diese Weise wird jene Korrelation der unmittelbar gegenwärtigen Vorstellung und der Gesammtvorstellung als des inaktualen Hintergrundes der ersteren genauer bestimmt.

4) Von Kants „Analogie der Erfahrung“ schließlich gewinnt Schopenhauer den Grundrahmen der, für seine Willenslehre entscheidenden und wichtigen „Analogie des Willens“. Aber auch hier erfolgt der Zugang nicht unmittelbar über Kant, sondern sehr wahrscheinlich vermittelt durch Schellings Ideen zu einer Philosophie der Natur (1797) und Von der Weltseele (1798), in denen der Begriff der Analogie mehrfach behandelt wird. Von diesen beiden Werken Schellings wissen wir, dass Schopenhauer sie sehr gründlich und wahrscheinlich sogar ursprünglich mit großer Begeisterung gelesen hatte. Schellings Verständnis von Analogie fand der junge Schopenhauer mit der Zeit jedoch zu unkritisch und zu sehr spekulativ. So greift schopenhauer auch hier wieder auf Kant zurück. Trotz des unterschiedlichen Analogie-Verständnisses lehnt sich Schopenhauer dennoch in seiner analogen Beschreibung der Natur weitgehend an Schellings Potenzenlehre an, die ihrerseits die abendländische Tradition der analogia entis auf ihre ganz eigene Weise weiterführt, so dass oft der Eindruck entsteht, dass Schopenhauer hier Schellings Naturphilosophie einfach kopiert habe, wenn man den grundsätzlichen Unterschied zwischen den Analogie-Verständnissen Schopenhauers und Schellings unberücksichtigt läßt. 
Schopenhauers Rückgang auf Kants Analogiebegriff bedeutet nun, dass der Begriff der Substanz etwa, nicht mehr als das an sich Seiende im abendländischmetaphysischen Sinne verstanden werden kann.

Auch wenn im 2. Band der Welt als Wille und Vorstellung (1844) der Wille oft als Substanz des Menschen bzw. als Substanz der Welt bezeichnet wird, so bedeutet dies jedoch keinesfalls eine Substanz im traditionell-metaphysischen Sinne eines an sich Seienden, das hinter der Welt subsistierte, wie es Schopenhauers zeitgenössische und auch spätere Kritiker bis hin zu Nietzsche und auch seine treuen Schüler wie Frauenstädt missverstanden haben -, sondern lediglich als eine Substanz, wie sie in der Kantischen Analogie der Erfahrung bestimmt wird.

So schrieb Schopenhauer 1852 an Frauenstädt, dem er eine realistischmaterialistische Tendenz vorwarf und mit dem er sogar später brach, folgende Zeilen:

„In meinem Hauptwerk Bd. 2, p. 204. steht allerdings «der Wille ist die Substanz des Menschen»; aber dabei steht auch, daß dies «bildlich und gleichnißweise» zu verstehen sei.“ (Brief von Schopenhauer an Frauenstädt, 1852, Deussen-XV135)

In diesem Zusammenhang muss auch betont werden, dass Schopenhauers Verständnis von Materie dem der Substanz der Kantischen Analogie der Erfahrung entspricht, was der sonst für die Verbreitung der Schopenhauerschen Philosophie so verdienstvolle Frauenstädt, linkshegelianischer Provenienz, so leider nie gesehen hatte.

Kant selber erläutert in der „Analogie der Erfahrung“ den transzendentalphilosophischen Begriff der Substanz wie folgt: „Nun kann die Zeit für sich nicht wahrgenommen werden. Folglich muß in den Gegenständen der Wahrnehmung, d.i. den Erscheinungen, das Substrat anzutreffen sein, welches die Zeit überhaupt vorstellt, und an dem aller Wechsel oder Zugleichsein durch das Verhältnis der Erscheinungen zu demselben in der Apprehension wahrgenommen werden kann.“ (B 225) Ganz in diesem Kantischen Sinne führt der junge Schopenhauer in seiner Dissertation Ueber die vierfache Wurzel im $\$ 19$ den Begriff der Materie erstmals als Wahrnehmbarkeit von Raum und Zeit (Go §19) ein. Diese Definition wird weiter unten im $\S 42$ wie auch mehrfach im ersten Buch von Die Welt als Wille und Vorstellung wiederholt. 
In dem zweiten Teil meiner Ausführung werde ich aus Zeitgründen den letzten und vierten Punkt in das Zentrum meiner Betrachtungen rücken. Zwar habe ich auch diesen, wie schon die Punkte eins bis drei, in Der junge Schopenhauer von 1988, schon einmal abgehandelt, jedoch seinerzeit als immanente Interpretation und ohne ausdrückliche Bezugsnahme zu Kants „Analogie der Erfahrung“. Aber vorher kommt noch ein Zwischenspiel.

\section{Zwischenspiel}

$\mathrm{Zu}$ Beginn des Studiums der Philosophie des jungen Schopenhauers stand dessen religiös-metaphysische Sehnsucht nach dem ewig-unwandelbaren Sein im Vordergrund, die in „Duplizität des Bewußtseins“, nämlich des empirischen bzw., besseren Bewußtseins ihren Ausdruck fand. Mit der Vertiefung seiner philosophischen Studien, hier ist die Lektüre Kants, Fichtes und Schellings zu nennen, schlug diese anfänglich romantische Haltung radikal ins Gegenteil um, was wir in Ueber die vierfache Wurzel am deutlichsten beobachten können. Hier ist die Grundstruktur der Welt als Vorstellung und jene des transzendentalen Willens streng in ihrer Bewußtseinsimmanenz des Vorstellungs-Seins herausgearbeitet.

Im Jahre 1814 erarbeitete sich Schopenhauer die Konzeption der Platonischen Idee als Produkt von Einbildungskraft und Vernunft und dies nachdem er die Platonischen Ideen als „Normalanschauung“ schon in Ueber die vierfache Wurzel (Go, §40) in den Anmerkungen aufgegriffen hatte. Der Ausdruck „Normalanschauung“ wurde hierbei offenbar durch die ,ästhetische Normalidee“ in der Kritik der Urteilskraft insperiert, wie sein Studienheft bezeugt (HNII, 288).

Parallel hierzu erhält die Platonische Idee dann die Bezeichnung „Ding an sich“.

Der Begriff des „Dinges an sich“ wird hier von Schopenhauer nicht mehr als die hinter der Erscheinung an sich seienden Substanz verstanden, wie er noch gegen den Kantischen Begriff des Dings an sich polemisiert hatte. Der Begriff Ding an sich wird nun für das Prinzip, das die Einheit und Stabilität der Erkenntnis der Welt als Vorstellung gewährleistet, verwendet. Er ist somit ein regulatives Prinzip, das die Bedingung der Möglichkeit der Welt als Vorstellung und deren Erkennbarkeit in ihrer Ganzheit ausmacht. 
Die Platonische Idee ist nicht wie einzelne anschauliche Vorstellungen durch Zeit, Raum und Kausalität an die einzelnen Erscheinungen gebunden, oder, um es in Schopenhauers Terminologie nach $1815 \mathrm{zu}$ formulieren, also $\mathrm{zu}$ einem Zeitpunkt, ab welchem der Gedanke der Analogie des Willens für Schopenhauer mittlerweile feststand, dem principium individuationis nicht unterworfen. Sie transzendiert in gewisser Weise so die einzel-anschaulichen Vorstellungen, weshalb sie von ihm Ding an sich genannt wurde.

Auch der Gedanke des Willens als Ding an sich wurde zwar bereits im Jahr 1814, kurz nach seinem Umzug von Weimar nach Dresden von ihm zum Ausdruck gebracht.

„Die Welt als Ding an sich ist ein großer Wille, der nicht weiß was er will.“ (HNI, 169 §278, Dresden 1814.)

Hier weist der Ausdruck, ,großer Wille“ über die in Uber die vierfache Wurzel festgehaltenen transzendentalphilosophischen Grundrahmen des Selbstbewußtseins hinaus und auf einen Willen als „das Weltschaffende“ hin.

Schopenhauer scheint damit auch das Fundament seiner Naturphilosophie im 2. Buch der Welt als Wille und Vorstellung vorwegzunehmen.

Indes dieser Gedanke des Willens als Weltschaffender bleibt dennoch eine Zeitlang ein reiner Einfall Schopenhauers. Weder in der zeitaktuellen Aufzeichnung noch in jenen davor oder danach wird eine nähere philosophische Erläuterung hierzu gegeben. Diese Aufzeichnung dürfte vielmehr einen Gedanken Friedrich Majers (1771-1818) widerspiegeln, eines Indologen und HerderSchülers, den Schopenhauer im Salon seiner Mutter Johanna Schopenhauer in Weimar kennengelernt hatte. Schopenhauer war offensichtlich von diesem Gedanken beeindruckt und konnte ihn mit seinem eigenen Lebensgefühl in Verbindung bringen, ohne ihn jedoch in dem in Ueber die vierfache Wurzel angelegten philosophischen Kontext stringent eingliedern zu können. In der Tat findet sich kurz vor der oben genannten Aufzeichnung eine in diesem Kontext ebenso interessante: „Aber der böse Wille in ihm wird nicht durch den Tod getilgt, sondern nur eine einzelne Erscheinung desselben, jener Leib der stirbt. Jener Wille lebt durch alle Zeit, denn er ist ihr wesentlich, wie sie ihm. Die Millionen Jahre der steten Wiedergeburt bestehn bloß im Begriff, (wie die ganze Vergangenheit und Zukunft bloß im Begriff besteht) für das Individuum ist die Zeit immer neu, es 
Kamata, Y. Schopenhauer und Kant. Rezeption de „transzendentalen Analytik“ der Kritik der reinen Vernunft in der Frühphilosophie Schopenhauers

findet sich stets als neu geschaffen: die erfüllte Zeit ist lauter Gegenwart.“ (HNI,161 §263, Dresden 1814)

In seiner Dresdner Zeit des Jahres 1814 entstand das Grundgerüst von Schopenhauers Theorie der Kunst und des Künstlers aufgrund der Begriffsbestimmung der Platonischen Idee als Phantasma. Auch der Gedanke der Verneinung des Willens nahm allmählich Kontur an. Ich möchte hier lediglich die zwei wichtigen Bausteine der Willensverneinung nennen: 1.) Seine veränderte Lebensauffassung, vor allem der Gedanke der Vergänglichkeit allen innerweltlichen Seins insbesondere des hinfälligen Lebens und des gleichzeitig immer stärker werdenden Bewußtseins, dass es keine religiös-metaphysische Erlösung jenseits der Welt gebe. Diese Veränderung in seiner Lebensauffassung ist für den mehrfachen Bedeutungswandel seines ursprünglichen Verständnis vom besseren Bewußtseins und die deutlicher werdende Entmetaphysizierung verantwortlich, bis das bessere Bewußtsein keine eigenständige Existenzberechtigung mehr hatte und sich in dem Gedanken der Verneinung des Willens auflöste. 2.) Der Sturz des ancient regime und der Zusammenbruch der Französischen Revolution in dem Terror der Diktatur, parallel zum Sturz der Substanzmetaphysik als ein philosophisches ancient regime und die Katastrophe der philosophischen Revolution in der Gestalt des Idealismus, allem voran der Fichteschen Philosophie in ihrer willkürlichen, substanzlosen Spekulation - später spricht Schopenhauer sehr deutlich von dem deutschidealistischen Windbeutel und dem Scharlatan.

Während die Herausbildung der Ästhetik und der Ethik allmählich fortschritt, blieb die Grundlegung der Naturphilosophie auf der Stelle stehen. Dank seines Umganges mit namhaften Physiologen und Biologen seiner Zeit, namentlich Blumenbach in Göttingen und Lichtenstein in Berlin, verfügte Schopenhauer über sehr gute zeitaktuelle naturwissenschaftliche Kenntnisse. Sie konnten jedoch nicht in seinem philosophischen Grundgerüst integriert werden. Mit dieser letzten Frage, wie denn nun die Natur in dem System des transzendentalen Idealismus einzuholen sei, die Frage, die jedem Philosophen des deutschen Idealismus zum Stein des Anstoßes wurde, musste Schopenhauer sich auch auseinandersetzen. Die Analogie des Willens war hierbei die Schopenhauersche Antwort auf eben diese Frage, zu der er gelangte, indem er von dem deutschen Idealismus auf Kant, namentlich auf dessen Analogie der Erfahrung zurückgriff. 
Kamata, Y. Schopenhauer und Kant. Rezeption de „transzendentalen Analytik“ der Kritik der reinen Vernunft in der Frühphilosophie Schopenhauers

Im Folgenden wollen wir nun den Gedankenprozeß des jungen Schopenhauer $\mathrm{zu}$ rekonstruieren versuchen, wie er jene Gleichsetzung der Platonischen Idee mit dem Ding an sich verwarf und statt dessen, über die Rezeption des Kantischen Analogiebegriffs, insbesondere der Analogie der Erfahrung, zu der Gleichsetzug von Wille und Ding an sich gelangte, ohne hierbei die transzendentalphilosophische Ebene $\mathrm{zu}$ verlassen. In vielen bisherigen Interpretationsansätzen $\mathrm{zu}$ Schopenhauer wurde oft als selbstverständlich angenommen, dass das Ding an sich so etwas wie die hinter der Erscheinungswelt an sich seiende Substanz sei und die Willenslehre Schopenhauers auf eine metaphysisch-ontologische All-Einheitslehre hinausliefe. So ist man in die Zwickmühle eines selbsterzeugten Widerspruchs hineingeraten.

\section{Von der Kantischen Erfahrung der Analogie zur Schopenhauerschen Analogie des Willens}

Wie wir bisher gesehen haben, hat der junge Schopenhauer bereits in Ueber die vierfache Wurzel vierschiedene Argumente aus der Kantischen „Analytik der Grundsätze" rezipiert und für seine Philosophie umgearbeitet. Es läßt sich allerdings nicht feststellen, dass er hierbei die charakteristische Denkweise Kants mit $\mathrm{zu}$ rezipieren gedachte, die die Kantische „Analogie der Erfahrung“ durchdringt.

Als sich die Frage mit dem Jahre 1814, wie denn die Philosophie der Natur auf transzendentalphilosophischem Boden gepflanzt werden könnte, immer weiter in den Vordergrund drängte, schaute sich Schopenhauer hierfür, was auf der Hand liegt, zuerst einmal bei Schelling um, der als prominenter Vertreter eben dieser Frage galt, und stieß bei diesem auf den Begriff der Analogie, wie wir bereits gesehen haben. Dieser Umweg führte Schopenhauer schließlich zur Kantischen „Analogie der Erfahrung“. Demgegenüber gibt es noch eine zweite, wichtige Entwicklungslinie dieses Analogieverständnisses, namentlich die Analogie in der Kritik der Urteilskraft, mit der sich Uenoyama, mein Schüler in Japan, beschäftigt.

Im Jahr 1815 schrieb Schopenhauer: „Der Wille ist Kants Ding an sich: und die Platonische Idee ist die völlig adäquate und erschöpfende Erkenntniß des Dings an sich, ist der Wille als Objekt.“ (HNI,291 §442). Hier ist zwar der Ausdruck der Analogie nicht explizit zu finden, jedoch unmittelbar nach der eben zitierten Stelle spricht der junge Schopenhauer von der subsumierenden Urteilskraft, womit „der ethic@-Florianópolis, v. 11, n. 1, p. 54-67, julho de 2012. 
Kamata, Y. Schopenhauer und Kant. Rezeption de „transzendentalen Analytik“ der Kritik der reinen Vernunft in der Frühphilosophie Schopenhauers

einzelne Fall mehreren Begriffen subsumiert wird“ (NHI, 299 §454). Das ist eine starke Bezugnahe zu Stellen in der Kritik der reinen Vernunft B 176 und B 304, die ich hier aus Zeitgründen nicht mehr zitieren kann.

Fünf Jahre später vermerkt Schopenhauer in seiner Berliner Vorlesung: „Diese (=Analogie, YK) ist das Werk der subsumierenden Urtheilskraft: denn sie geht darauf aus, einen einzelnen Fall oder Gegenstand unter eine schon bekannte Regel oder Begriff zu subsumieren und folglich sodann diesem gemäß zu beurtheilen.“ Diesem Zitat können wir nicht nur entnehmen, dass Schopenhauers Analogie-Verständnis sich nach dem Kantischen richtet, sondern auch, dass er ebenso nach der - und deshalb auch regelrecht während der - Abfassung der Welt als Wille und Vorstellung an diesem Verständnis festhielt.

$\mathrm{Ab}$ jetzt werden die Analogie des Willens einerseits und die Gleichsetzung von Wille und Ding an sich andererseits parallel gebildet und weitergeführt. Denn das Ding an sich bedeutet in dieser Phase seines Denkens, wie wir oben nachgewiesen haben, kein hinter der Erscheinungswelt an sich subsistierendes Seiendes, sondern ist als ein analoger Begriff wie die Substanz in der Kantischen Analogie der Erfahrung zu verstehen.

Wie versteht nun aber Kant die Analogie? Kant unterscheidet zwischen dem Analogie-Verständnis der Mathematik und jener der Philosophie.

Die Formeln der mathematischen Analogie, sie wird auch als Proportion übersetzt, ist jederzeit konstitutiv, so dass „wenn drei Glieder der Proportion gegeben sind, auch das vierte dadurch gegeben wird, d.i. construirt werden kann“" (B 222). Die philosophische Analogie ist kein konstitutives, sondern lediglich ein regulatives Prinzip, um das vierte Glied zu suchen, das sich nach dem gegebenen Gesetz mit den vorhergehenden drei Gliedern verträgt. Deshalb ist die philosophische Analogie nicht im Stande, über die Dinge, die außerhalb der Erfahrung, d.h. außer der Welt als Vorstellung subsistieren, etwas auszusagen.

Um die Vielfalt der Eindrücke und deren anfängliche Apprehension in der Anschauung, die ihrer Seinsart nach gänzlich verschieden ist von der durch die Apperzeption verwalteten Einheit des Begriffssystems, eben als durch diese begreifbar nachzuweisen, müssen die einzelnen Anpassungsschritte mit der daran beteiligten Erkenntnisfunktion aufgezeigt werden. Das ist die Stringenz des Denkens, die Kant von der Philosophie verlangt - und ebenso der junge Schopenhauer, der Verfasser von Ueber die vierfache Wurzel. 
Diese Schritte zeigt Kant mittels seines Schematismus und der daran beteiligten Erkenntnisfunktion als subsumierendes Urteilskraft. Die raum-zeitlich aufgefasste Mannigfaltigkeit der Eindrücke der Anschauung wird nämlich gemäß den Grundregeln des Denkens, d.h. mit den Begriffen Substanz, Kausalität und Wechselwirkung, zusammengefasst, unter die synthetische Einheit der Apperzeption gebracht und von ihr verwaltet. Deshalb nennt Kant die Wirkungsweise des Verstandes Funktion, während er die der Sinnlichkeit Affektion nennt.

Insofern ist die Erfahrung im Sinne der empirischen Erkenntnis durch Analogie von Substanz, Kausalität und Wechselwirkung die einzig mögliche Weise, in der uns die Gegenstände der Erfahrung gegeben und begriffen werden können und deren objektive Realität nur innerhalb der Erfahrung, d.h. die empirische Realität der Erscheinung gewährleistet wird.

Wenn man nun aber bedenkt, dass diese Erfahrung nur solange existiert, als sie innerhalb der Bedingungen der Möglichkeit der Erfahrung verweilt und umgekehrt ohne deren Annahme zumindest in dieser Seinsweise nicht existieren kann, so erkennen wir die transzendentale Idealität der Erscheinung.

Eben deshalb ist die „Analogie der Erfahrung“ weder berechtigt noch im Stande, etwas über die metaphysisch an sich seiende Substanz auszusagen, wovon alle andere sinnlich-endliche Dinge hergeleitet werden könnten.

Im folgenden möchte ich die Sätze aus Schopenhauers Handschriftlichem Nachlaß zitieren, mit denen er die „Analogie des Willens“ zum ersten Mal formuliert. Schopenhauer schreibt hier: „der Wille, aus dem die Welt ist, offenbart sich in jeder Muskelbewegung so daß er keinem Zweifel Raum läßt: jeder weiß, was meine Hand bewegt ist Wille.“ (HNI,390 §575) Hier wird also nicht der große Wille thematisiert, der bei der Entstehung der Welt gewirkt hätte, sondern der Wille im Sinne der Spontaneität, der im Kapitel 7 von Ueber die vierfache Wurzel in dem transzendentalphilosophischen Zusammenhang abgehandelt wurde. Er fährt dann fort: „Von allen Dingen die nicht ich selbst sind, erkenne ich bloß eine Seite, die der Vorstellung: ihr innres Wesen ist mir völlig geheimnißvoll, auch dann wann ich alle Ursachen weiß auf welche ihre Veränderungen erfolgen. Nur aus der Vergleichung mit dem was in mir vorgeht, wenn ich eine Aktion ausübe, und wie diese auf ein Motiv erfolgt, kann ich nach der Analogie verstehn, wie jene todten Körper sich auf Ursachen verändern, und was ihr innres Wesen sei, von dessen ethic@-Florianópolis, v. 11, n. 1, p. 54-67, julho de 2012. 
Erscheinung nichts als die bloße Zeitfolge aus den äußern Ursachen erklärlich ist. Dies kann ich darum, weil ich selbst, weil mein Leib, das einzige ist, davon ich auch die 2te Seite erkenne, welche ich Wille nenne a potiori.“ (HNI,390 §575)

Es handelt sich somit hier lediglich um die analoge Benennung der unbekannten Bedingung der Möglichkeit der Welt als Vorstellung nach dem mir unmittelbar bekannten transzendentalen Willen, wodurch nicht nur die Relationen der toten Dinge, sondern auch das Verständnis der lebendigen Wesen und insbesondere anderer Menschen möglich wird. Nirgendwo findet man hier die großartige Verkündigung eines Weltwillens im Sinne eines metaphysischen Weltschaffenden hinter der Welt als Vorstellung.

In der aus vermutlich gleicher Zeit stammenden Notiz Schopenhauers über Kant heißt es, „Statt dessen hätte K[ant] unmittelbar vom Willen ausgehn[,] ihn als das unmittelbar erkannte Ansich unsrer eignen Erscheinung nachweisen.“ (HNII,421. Gegen Kant).

Hier zeigt sich besonders deutlich, dass der Ausdruck „an sich“ „dem Satz vom Grunde nicht unterworfen" meint und deshalb genauso wenig eine substanzmetaphysische Bedeutung besitzt wie der Begriff Aseität, der in Wille in der Natur (1836) und in den späteren Werken zu finden ist. Zum Begriff Aseität verweise ich auf die Untersuchung von Yoichiro Takahashi, dem jetzigen Schriftführer der japanischen Schopenhauer-Gesellschaft: „Die Aseität des Willens - Freiheitslehre Schopenhauers in seiner mittleren und späteren Phase“ (1996, japanisch).

Der oben angeführte Gedanke wird dann an die Stelle der Analogie des Willens in DieWelt als Wille und Vorstellung aufgenommen.

„Wir werden demzufolge die ... auf zwei völlig heterogene Weisen gegebene Erkenntniß, ..., weiterhin als einen Schlüssel zum Wesen jeder Erscheinung in der Natur gebrauchen und alle Objekte, ... eben nach Analogie jenes Leibes beurtheilen und daher annehmen, daß, ... das dann noch übrig Bleibende, seinem innern Wesen nach, das selbe seyn muß, als was wir an uns Wille nennen.“ WI, $125(\S 19)$

Der hier verwendete Ausdruck „(subsumierend) beurteilen und daher annehmen“ läßt uns, wie die Formulierung im Handschriftlichen Nachlaß nahelegt, unterbindet jedwede metaphysisch-substantialistische Ambition nach einer mathematisch eindeutigen Analogie im Sinne einer proportio. 
Kamata, Y. Schopenhauer und Kant. Rezeption de „transzendentalen Analytik“ der Kritik der reinen Vernunft in der Frühphilosophie Schopenhauers

Eine derartige Interpretation im Sinne einer mathematischen Proportion begann bereits mit Fortlages Besprechung der Welt als Wille und Vorstellung im Jahre 1844, sie wurde fortgeführt von Frauenstädt, Volkert und anderen namhaften Schopenhauer-Forschern, und heute von Ekkehardt Zimmermann in seiner Dissertation von 1970, aus welcher so mancher Philosophiehistoriker kritiklos abschrieb. So etablierte sich eine "Theorie des Analogieschlusses des Willens" quasi selbstständig und noch schlimmer, in aller Selbstverständlichkeit.

Bisher wurde an der so missverstandenen Schopenhauerschen „Analogie des Willens“ mehrfach Kritik geübt. In der Selbstverständlichkeit der problematischen Interpretation der als mathematische Proportion verstandenen Analogie des Willens erschien der logische Nachweis des Willens als ein substantialistisch verstandener Weltwille natürlich eingänglich, einfach aber eben auch oberflächlich. Eine solche Erklärung kommt jenem zupass, der die Schopenhauersche Philosophie als Pseudoreligion und populäre Weltanschauung verstanden wissen möchte oder sie als solche abtun will, die Rezeptionsgeschichte ist hier voll an Beispielen. Wer die Analogie des Willens derart als mathematische Proportion vereinfacht, wird gerade eine ins Auge springende Oberflächlichkeit der Interpretation enttäuscht, die er dann bei der nächstbesten Gelegenheit der Philosophie Schopenhauers selbst zuschreibt.

Die Folgen sind bis in die Aktualität hinein gravierend.

Ich möchte Sie hierbei lediglich auf einen wichtigen Punkt aufmerksam machen.

Durch die Analogie des Willens steht die empirische Realität der Erscheinung im Kantischen Sinn fest. Das bedeutet, dass man, so lange man innerhalb der Erscheinung bleibt und sich einem keine übernatürlichmetaphysische Frage stellt, im Alltag ganz von dem transzendentalen Aspekt der Erscheinung, nämlich von deren transzendentaler Idealität in Bezug auf das Subjekt des Erkennens und des Wollens absehen kann. Das bedeutet nun, dass man insofern als reiner Realist, reiner Materialist denken kann, wobei der Wille selbst als ein Lebensprinzip in der Natur bzw. als der blinde Wille zum Leben erscheint, oder eben als reiner Idealist leben kann, wobei der Wille als die die Welt als Vorstellung verändernde praktische Fähigkeit aufgefasst werden kann, als man die transendentalphilosophische Fragedimension hierbei nicht vergisst. So formulierte Schopenhauer auch bereits in Ueber die vierfache Wurzel wie folgt: „Demnach ist ethic@-Florianópolis, v. 11, n. 1,p.54-67,julho de 2012. 
Kamata, Y. Schopenhauer und Kant. Rezeption de ",transzendentalen Analytik“ der Kritik der reinen Vernunft in der Frühphilosophie Schopenhauers

es ganz einerlei, ob ich sage: Die Objekte haben solche und solche ihnen anhängende und eigenthümliche Bestimmungen; oder: das Subjekt erkennt auf solche und solche Weisen: einerlei ob ich sage: die Objekte sind in solche Klassen zu theilen; oder: dem Subjekt sind solche unterschiedne Erkenntnißkräfte eigen.“ (Go, $70 \S 42)$

Wenn man aber diese transzendentale Grundlage außer acht läßt, und in den einfachen Positivismus des Machbaren versinkt, besteht die Gefahr, die Gemeinschaftlichkeit des Menschen zu vernichten, denn die Eigennützigkeit des modernen homo oeconomicus, als eine Fügung von Eigen und Nutz, stellt einen diametralen Gegensatz zur Fügung von Mit und Leiden, Mitleiden dar.

Der unaufhaltsame Fortschritt der Naturwissenschaften prägte verstärkt den Zeitgeist des 19. Jahrhunderts, und dies Hand in Hand mit einer unaufhaltsamen Entwicklung und Erweiterung der Ökonomie und des Kolonialismus. Schopenhauer war, bewußt oder unbewußt, gezwungen, seine Philosophie in immer neuen Wendungen und Bildern $\mathrm{zu}$ präsentieren. In dieser Folge nahm der Anteil der naturwissenschaftlichen bzw. naturphilosophischen Darstellungen $\mathrm{zu}$, die bei Schopenhauer eigentlich nur auf der Ebene der „Analogie des Willens“ zu verstehen sind. So entstand der Anschein, daß eine realistische bzw. materialistische Wende der Schopenhauerschen Philosophie stattgefunden hätte, von der selbst treue Schüler wie Frauenstädt, welche der Annahme folgten, getäuscht waren, dass es letztlich zum Bruch mit dem Meister kam und erst der Tod Schopenhauers diese versöhnte. 